\title{
EXISTENCE OF WEAK SOLUTIONS FOR ELLIPTIC SYSTEMS WITH $p, q$-GROWTH
}

\section{Giovanni Cupini, Francesco Leonetti and Elvira Mascolo}

\author{
Università di Bologna, Dipartimento di Matematica \\ Piazza di Porta S. Donato 5, 40126 Bologna, Italy; giovanni.cupini@unibo.it \\ Università di L'Aquila, Dipartimento di Ingegneria e Scienze dell'Informazione, Matematica \\ 67100 L'Aquila, Italy; leonetti@univaq.it \\ Università di Firenze, Dipartimento di Matematica e Informatica "U. Dini" \\ Viale Morgagni 67/A, 50134 Firenze, Italy; mascolo@math.unifi.it
}

\begin{abstract}
We consider a non-linear system of $m$ equations in divergence form and a boundary condition:

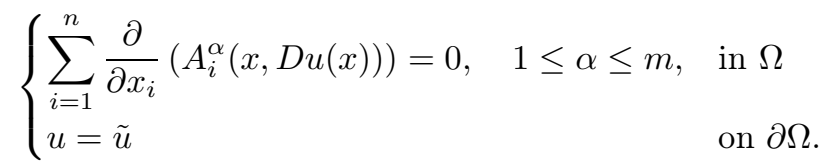

The functions $A_{i}^{\alpha}(x, z)$ are Hölder continuous with respect to $x$ and

$$
|z|^{p}-c_{1} \leq \sum_{\alpha=1}^{m} \sum_{i=1}^{n} A_{i}^{\alpha}(x, z) z_{i}^{\alpha} \leq c_{2}(1+|z|)^{q}, \quad 2 \leq p \leq q .
$$

We prove the existence of a weak solution $u$ in $\left(\tilde{u}+W_{0}^{1, p}\left(\Omega ; \mathbf{R}^{m}\right)\right) \cap W_{\text {loc }}^{1, q}\left(\Omega ; \mathbf{R}^{m}\right)$, provided $p$ and $q$ are close enough and under suitable summability assumptions on the boundary datum $\tilde{u}$.
\end{abstract}

\section{Introduction}

In this paper we are concerned with the existence and regularity of solutions to the Dirichlet problem associated to a non-linear system of $m$ equations in divergence form

$$
\begin{cases}\sum_{i=1}^{n} \frac{\partial}{\partial x_{i}}\left(A_{i}^{\alpha}(x, D u(x))\right)=0, \quad 1 \leq \alpha \leq m, & \text { in } \Omega \\ u=\tilde{u} & \text { on } \partial \Omega,\end{cases}
$$

where $\Omega$ is a bounded open set in $\mathbf{R}^{n}, n \geq 2$, and $\tilde{u}$ is a vector-valued function in a suitable Sobolev space.

We assume that $A_{i}^{\alpha}: \Omega \times \mathbf{R}^{n m} \rightarrow \mathbf{R}, 1 \leq i \leq n, 1 \leq \alpha \leq m$, are Carathéodory functions satisfying the following properties: there exist $2 \leq p \leq q, 0<\gamma \leq 1$ and $\nu, M, c>0$, such that for every $x, y \in \Omega$ and for every $z, \tilde{z} \in \mathbf{R}^{n m}$ :

doi:10.5186/aasfm.2015.4035

2010 Mathematics Subject Classification: Primary 35J25; Secondary 35J47, 49N60.

Key words: Existence, regularity, weak, solution, elliptic, system, growth.

Acknowledgement: We would like to thank Dominic Breit for raising a question that led us to the present study. The authors have been supported by the Gruppo Nazionale per l'Analisi Matematica, la Probabilità e le loro Applicazioni (GNAMPA) of the Istituto Nazionale di Alta Matematica (INdAM). 
(H1) $\nu\left(|z|^{2}+|\tilde{z}|^{2}\right)^{\frac{p-2}{2}}|z-\tilde{z}|^{2} \leq \sum_{\alpha=1}^{m} \sum_{i=1}^{n}\left[A_{i}^{\alpha}(x, z)-A_{i}^{\alpha}(x, \tilde{z})\right]\left[z_{i}^{\alpha}-\tilde{z}_{i}^{\alpha}\right]$,

(H2) $\left|A_{i}^{\alpha}(x, z)\right| \leq M(1+|z|)^{q-1}$,

(H3) $\sum_{\alpha=1}^{m} \sum_{i=1}^{n}\left|A_{i}^{\alpha}(x, z)-A_{i}^{\alpha}(y, z)\right| \leq c|x-y|^{\gamma}(1+|z|)^{q-1}$.

Notice that we are in the framework of the $p, q$-growth, since (H1) and (H2) imply that there exist $c_{1}, c_{2}>0$ such that

$$
\frac{\nu}{2}|z|^{p}-c_{1} \leq \sum_{\alpha=1}^{m} \sum_{i=1}^{n} A_{i}^{\alpha}(x, z) z_{i}^{\alpha} \leq c_{2}(1+|z|)^{q} .
$$

For $\tilde{u} \in W^{1, q}\left(\Omega ; \mathbf{R}^{n m}\right)$, a weak solution $u$ to problem (1.1) is a function $u$ such that

$$
u-\tilde{u} \in W_{0}^{1, p}\left(\Omega ; \mathbf{R}^{m}\right) \cap W_{\mathrm{loc}}^{1, q}\left(\Omega ; \mathbf{R}^{m}\right)
$$

and

$$
\int_{\Omega} \sum_{\alpha=1}^{m} \sum_{i=1}^{n} A_{i}^{\alpha}(x, D u) \varphi_{x_{i}}^{\alpha}(x) d x=0 \quad \forall \varphi \in W^{1, q}\left(\Omega ; \mathbf{R}^{m}\right), \operatorname{supp} \varphi \Subset \Omega .
$$

If $p=q$, the existence of weak solutions to (1.1) can be established using the theory of coercive, monotone operators, see Leray-Lions [24], Browder [4] and HartmanStampacchia [20]. Moreover, by (H1) the solution is unique. Also the regularity issue has been extensively studied, see the monographs [18], [19] and the surveys [28] and [29].

If $p<q$ the above classical existence results cannot be applied due to the lack of coercivity in $W^{1, q}$. Moreover, the request $u \in W_{\text {loc }}^{1, q}\left(\Omega ; \mathbf{R}^{m}\right)$ in the definition of weak solution, needed to have a well defined integral in (1.4), is an additional difficulty. Notice that such a request is a priori assumed in some regularity results under the $p, q$-growth, see for example Leonetti [22], Bildhauer-Fuchs [1] and CupiniMarcellini-Mascolo [10].

In this paper we prove the existence of a weak solution $u \in W_{\mathrm{loc}}^{1, q}\left(\Omega ; \mathbf{R}^{m}\right)$ to (1.1). Moreover, under additional assumptions, we show that $u$ is locally bounded.

The existence of weak solutions is proved assuming that the integrability of the boundary datum is high enough. Precisely, our result is the following.

Theorem 1.1. Assume that (H1)-(H3) hold, with

$$
2 \leq p \leq q<p \frac{n+\gamma}{n} .
$$

For every $\tilde{u} \in W^{1, p \frac{q-1}{p-1}}\left(\Omega ; \mathbf{R}^{m}\right)$ there exists a weak solution $u$ to the Dirichlet problem (1.1), that is $u$ satisfies (1.3) and (1.4). Moreover, $u \in W_{\text {loc }}^{1, s}\left(\Omega ; \mathbf{R}^{m}\right)$ for all $1 \leq s<$ $p \frac{n}{n-\gamma}$.

As far as the regularity of solutions is concerned, the obstructions are essentially two: we are dealing with systems (if $m \geq 2$ ) and under non-standard growth $(p<q)$. Indeed, in the vectorial case, even under the standard growth, the everywhere regularity of solutions for systems, or of minimizers of integrals, cannot be expected unless some structure conditions are assigned, and this holds also for the local boundedness, see e.g. the counterexamples by De Giorgi [13] and Sverák-Yan [30]. 
Since the pioneering paper by Marcellini [26], the theory of regularity in the framework of non-standard growth has been deeply investigated. The results and the contributions to regularity are so many, that it is a hard task to provide a comprehensive overview of the issue. For this, we refer to the survey of Mingione [28] for an accurate and interesting account on this subject. A common feature is that to get regularity results $p$ and $q$ must be not too far apart, as examples of irregular solutions by Giaquinta [17], Marcellini [25], [27] and Hong [21] show. Notice that the rate of Hölder continuity of $A(\cdot, z)$ interacts with the ratio $q / p$ precisely as in $(1.5)$, see Esposito-Leonetti-Mingione [15] and Colombo-Mingione [8] where the same bound appears. This is suggested by an example in [15], where the minimizer of a functional with $q / p>1+\gamma / n$ fails to be locally $W^{1, q}$-regular; see also Fonseca-Malý-Mingione [16]. Moreover, the condition on the distance between the exponents $p$ and $q$ can usually be relaxed if the solutions/minimizers are assumed locally bounded, see e.g. Choe [7], D'Ottavio [14], Bildhauer-Fuchs [2], Canale et al. [5], Carozza-KristensenPassarelli [6], Breit [3], Colombo-Mingione [9] and Cupini-Marcellini-Mascolo [12].

A particular case of systems such that its weak solutions in $W_{\text {loc }}^{1, q}\left(\Omega ; \mathbf{R}^{m}\right)$ are locally bounded is given by

$$
\sum_{i=1}^{n} \frac{\partial}{\partial x_{i}}\left(\sum_{j=1}^{n} a_{i j}(x, D u) u_{x_{j}}^{\alpha}\right)=0, \quad \alpha=1, \ldots, m .
$$

This result is a consequence of Theorem 4.1 in [10], see Theorem 5.1 below. See also Leonetti-Mascolo [23] and Cupini-Marcellini-Mascolo [11].

Collecting the existence result Theorem 1.1 and Theorem 5.1, we obtain the existence of a locally bounded weak solution to (1.1) under the structure condition (1.6), see Theorem 5.2. We emphasize that no boundedness condition is imposed on the boundary datum $\tilde{u}$. Theorem 5.2 can be applied to the Euler equation of the functionals

$$
\int_{\Omega}\left(|D u(x)|^{2}+a(x)|D u(x)|^{q}\right) d x
$$

and

$$
\int_{\Omega}\left(1+|D u(x)|^{2}\right)^{\frac{p(x)}{2}} d x
$$

as described at the end of paper.

The plan of the paper is briefly described. In Section 2 we give the strategy of the proof of the existence result Theorem 1.1, which relies on an approximation argument, and state useful preliminary results. The a priori estimates needed to let the approximation work are proved in Section 3. The conclusion of the proof of Theorem 1.1 is in Section 4.

In the last section, we will apply the existence result to a particular class of nonlinear systems, see (5.3), and we will prove that for this class there exists a locally bounded weak solution of the corresponding Dirichlet problem, see Theorem 5.2. Applications of our results are given.

\section{Preliminary results}

We consider a bounded open set $\Omega$ in $\mathbf{R}^{n}, n \geq 2$, and Carathéodory functions $A_{i}^{\alpha}: \Omega \times \mathbf{R}^{n m} \rightarrow \mathbf{R}$, with $i=1, \ldots, n$ and $\alpha=1, \ldots, m$, satisfying (H1), (H2), (H3), with $2 \leq p \leq q, 0<\gamma \leq 1$ and $\nu, M, c>0$. 
Fixed $\tilde{u} \in W_{\text {loc }}^{1, q}\left(\Omega ; \mathbf{R}^{m}\right)$ we want to study the existence and the summability properties of the solutions to the elliptic system (1.1) in a weak sense; precisely, $u$ is a weak solution to (1.1) if $u-\tilde{u} \in W_{0}^{1, p}\left(\Omega ; \mathbf{R}^{m}\right) \cap W_{\text {loc }}^{1, q}\left(\Omega ; \mathbf{R}^{m}\right)$ satisfies (1.4).

For the sake of clarity, we report the scheme of the proof of Theorem 1.1. We approximate the system in (1.1) with the systems

$$
\sum_{\alpha=1}^{m} \sum_{i=1}^{n} \frac{\partial}{\partial x_{i}}\left(A_{\epsilon, i}^{\alpha}(x, D u)\right)=0, \quad 0<\epsilon<1,
$$

where $A_{\epsilon, i}^{\alpha}: \Omega \times \mathbf{R}^{n m} \rightarrow \mathbf{R}$ is defined as

$$
A_{\epsilon, i}^{\alpha}(z):=A_{i}^{\alpha}(z)+\epsilon|z|^{q-2} z_{i}^{\alpha} .
$$

By $(1.2), A_{\epsilon, i}^{\alpha}(x, z)$ satisfies

$$
\sum_{\alpha=1}^{m} \sum_{i=1}^{n} A_{\epsilon, i}^{\alpha}(x, z) z_{i}^{\alpha} \geq \epsilon|z|^{q}-\lambda
$$

Moreover, by (H1) and (H2), we have that

$$
\left|A_{\epsilon, i}^{\alpha}(x, z)\right| \leq(M+1)(1+|z|)^{q-1}
$$

and, since $z \mapsto|z|^{q-2} z$ is a monotone operator,

$$
\nu\left(|z|^{2}+|\tilde{z}|^{2}\right)^{\frac{p-2}{2}}|z-\tilde{z}|^{2} \leq \sum_{\alpha=1}^{m} \sum_{i=1}^{n}\left[A_{\epsilon, i}^{\alpha}(x, z)-A_{\epsilon, i}^{\alpha}(x, \tilde{z})\right]\left[z_{i}^{\alpha}-\tilde{z}_{i}^{\alpha}\right] .
$$

The classical theory of monotone operators, see e.g. [24], [4] and [20], permits to conclude that there exists (and, by (H1), unique) $u_{\epsilon} \in \tilde{u}+W_{0}^{1, q}(\Omega)$ such that

$$
\int_{\Omega} \sum_{\alpha, i} A_{\epsilon, i}^{\alpha}\left(x, D u_{\epsilon}\right) \varphi_{x_{i}}^{\alpha} d x=0 \quad \forall \varphi \in W_{0}^{1, q}\left(\Omega ; \mathbf{R}^{m}\right)
$$

Then, we prove estimates in Lebesgue spaces for the gradient of the functions $u_{\epsilon}$, uniform with respect to $\epsilon$ : the norms of $D u_{\epsilon}$ are bounded in $L^{p}(\Omega)$ and in $L^{q}\left(\Omega^{\prime}\right)$, $\Omega^{\prime} \Subset \Omega$, see Propositions 3.1 and 3.2 , respectively.

We conclude by proving that there exists $u_{0} \in \tilde{u}+W_{0}^{1, p}(\Omega)$ such that $u_{0} \in W_{\text {loc }}^{1, q}(\Omega)$ and, up to subsequences, $D u_{\epsilon} \rightarrow D u_{0}$ strongly in $L_{\text {loc }}^{p}(\Omega)$. This will imply that $A_{\epsilon, i}^{\alpha}\left(x, D u_{\epsilon}\right)$ converges, up to subsequences, to $A_{i}^{\alpha}\left(x, D u_{0}\right)$ in $L_{\mathrm{loc}}^{1}(\Omega)$. Passing to the limit in the equation (2.5), with $\varphi \in W^{1, q}\left(\Omega ; \mathbf{R}^{m}\right)$ with compact support in $\Omega$, we conclude.

We here recall some known results. To prove that the $L^{p}$ norms of $D u_{\epsilon}$ are bounded with respect to $\epsilon$, see Lemma 3.1, we need the following inequality, see also [27].

Lemma 2.1. Assume that (H1) and (H2) hold. Let $A_{\epsilon, i}^{\alpha}$ be defined as in (2.2). Then there exists $c>0$ such that for all $0<\epsilon<1$, for all $x \in \Omega$ and for all $\xi, \eta \in \mathbf{R}^{n m}$

$$
|\xi|^{p} \leq c\left\{\sum_{\alpha, i} A_{\epsilon, i}^{\alpha}(x, \xi)\left(\xi_{i}^{\alpha}-\eta_{i}^{\alpha}\right)+(1+|\eta|)^{\frac{p(q-1)}{p-1}}\right\} .
$$


Proof. By (2.4),

$$
\begin{aligned}
|\xi|^{p} & \leq 2^{p-1}\left(|\xi-\eta|^{p-2}|\xi-\eta|^{2}+|\eta|^{p}\right) \leq c\left(\left(|\xi|^{2}+|\eta|^{2}\right)^{\frac{p-2}{2}}|\xi-\eta|^{2}+|\eta|^{p}\right) \\
& \leq \tilde{c}\left(\sum_{\alpha, i}\left(A_{\epsilon, i}^{\alpha}(x, \xi)-A_{\epsilon, i}^{\alpha}(x, \eta)\right)\left(\xi_{i}^{\alpha}-\eta_{i}^{\alpha}\right)+|\eta|^{p}\right), \quad \forall \xi, \eta \in \mathbf{R}^{n m} .
\end{aligned}
$$

By (H2) and the Young inequality,

$$
\tilde{c}\left|\sum_{\alpha, i} A_{\epsilon, i}^{\alpha}(x, \eta)\left(\xi_{i}^{\alpha}-\eta_{i}^{\alpha}\right)\right| \leq c(M+1)(1+|\eta|)^{q-1}|\xi-\eta| \leq \frac{1}{2}|\xi|^{p}+c(1+|\eta|)^{\frac{p(q-1)}{p-1}} .
$$

Thus, (2.6) follows.

To get that the $L^{q}$ norms of $D u_{\epsilon}$ are locally bounded with respect to $\epsilon$, see Proposition 3.2, we need technical results. For the reader's convenience we list them below. The first one is based on an iteration argument, see e.g. [19].

Lemma 2.2. Let $Z(t)$ be a bounded and non-negative function in the interval $[\rho, R]$. Assume that for $\rho \leq r<d \leq R$

$$
Z(r) \leq \theta Z(d)+\frac{A}{(d-r)^{\gamma}}+B
$$

with $A, B \geq 0$ and $0 \leq \theta<1$. Then

$$
Z(\rho) \leq c(\gamma)\left[\frac{A}{(R-\rho)^{\gamma}}+B\right]
$$

The remaining results are related to fine properties of the difference quotient; they can be found e.g. in [15]. We recall that, given a function $f: \mathbf{R}^{n} \rightarrow \mathbf{R}^{k}$, then $\tau_{s, h} f(x)=f\left(x+h e_{s}\right)-f(x)$, where $e_{s}$ is the $s$-th vector of the canonical basis in $\mathbf{R}^{n}$.

Lemma 2.3. If $0<\rho<R,|h|<R-\rho, 1 \leq t<+\infty, s \in\{1, \ldots, n\}$, and $G, D_{s} G \in L^{t}\left(B_{R}\right)$, then

$$
\int_{B_{\rho}}\left|\tau_{s, h} G(x)\right|^{t} d x \leq|h|^{t} \int_{B_{R}}\left|D_{s} G(x)\right|^{t} d x .
$$

Lemma 2.4. If $G: \mathbf{R}^{n} \rightarrow \mathbf{R}^{k}, G \in L^{2}\left(B_{R}\right), 0<R \leq 1$ and for some $\rho \in(0, R)$, $d \in(0,1), M>0, \eta: \mathbf{R}^{n} \rightarrow \mathbf{R}$ with $\eta \in C_{c}^{1}\left(\frac{B_{\frac{\rho+R}{2}}}{2}\right), 0 \leq \eta \leq 1$ in $\mathbf{R}^{n},|D \eta| \leq \frac{4}{R-\rho}$ in $\mathbf{R}^{n}, \eta=1$ on $B_{\rho}$,

$$
\sum_{s=1}^{n} \int_{B_{R}}\left|\tau_{s, h} G(x)\right|^{2} \eta^{2}(x) d x \leq M^{2}|h|^{2 d}
$$

for every $h$ with $|h| \leq \frac{R-\rho}{4}$, then $G \in W^{b, 2}\left(B_{\rho} ; \mathbf{R}^{k}\right) \cap L^{\frac{2 n}{n-2 b}}\left(B_{\rho} ; \mathbf{R}^{k}\right)$ for every $b \in(0, d)$ and

$$
\|G\|_{L^{\frac{2 n}{n-2 b}\left(B_{\rho}\right)}} \leq \frac{c}{(R-\rho)^{2 b+2 d+2}}\left(M+\|G\|_{L^{2}\left(B_{R}\right)}\right),
$$

where $c=c(n, k, b, d)$.

Lemma 2.5. For every $p>1$ and $G: B_{R} \rightarrow \mathbf{R}^{k}$, we have

$$
\left|\tau_{s, h}\left(\left(\mu^{2}+|G(x)|^{2}\right)^{\frac{p-2}{4}} G(x)\right)\right|^{2} \leq c(k, p)\left(\mu^{2}+|G(x)|^{2}+\left|G\left(x+h e_{s}\right)\right|^{2}\right)^{\frac{p-2}{2}}\left|\tau_{s, h} G(x)\right|^{2}
$$

for every $x \in B_{\rho}$, with $|h| \leq R-\rho$ and every $s=1, \ldots, n$, where $0 \leq \mu \leq 1$ and the constant $c=c(k, p)$ is independent of $\mu$. 


\section{Uniform estimates}

In this section we study the approximating systems (2.1). The properties of $A_{\epsilon, i}^{\alpha}$, $i=1, \ldots, n, \alpha=1, \ldots, m$, ensure the existence of a unique function $u_{\epsilon} \in \tilde{u}+W_{0}^{1, q}(\Omega)$ such that

$$
\int_{\Omega} \sum_{\alpha, i} A_{\epsilon, i}^{\alpha}\left(x, D u_{\epsilon}\right) \varphi_{x_{i}}^{\alpha} d x=0 \quad \forall \varphi \in W_{0}^{1, q}\left(\Omega ; \mathbf{R}^{m}\right) .
$$

In the following, $c$ is a positive constant that may vary from line to line.

First we claim that the $L^{p}$ norms of $D u_{\epsilon}$ are bounded with respect to $\epsilon$.

Proposition 3.1. Assume that (H1) and (H2) hold and consider $\tilde{u} \in W^{1, \frac{p(q-1)}{p-1}}(\Omega)$. For all $\epsilon$, let $u_{\epsilon} \in \tilde{u}+W_{0}^{1, q}(\Omega)$ satisfy (2.5). Then there exists a positive constant $c_{0}$, independent of $\epsilon$, such that

$$
\int_{\Omega}\left|D u_{\epsilon}\right|^{p} d x \leq c_{0} \int_{\Omega}(1+|D \tilde{u}|)^{\frac{p(q-1)}{p-1}} d x
$$

Moreover, $\left\|u_{\epsilon}-\tilde{u}\right\|_{W_{0}^{1, p}(\Omega)} \leq c$ with $c$ independent of $\epsilon$.

Proof. By (2.5) with test function $\varphi=u_{\epsilon}-\tilde{u}$, and using Lemma 2.1, with $\xi=D u_{\epsilon}(x), \eta=D \tilde{u}(x)$, we get (3.1). Moreover, since

$$
\int_{\Omega}\left|D u_{\epsilon}-D \tilde{u}\right|^{p} d x \leq 2^{p-1} \int_{\Omega}\left(\left|D u_{\epsilon}\right|^{p}+|D \tilde{u}|^{p}\right) d x
$$

then (3.1) implies the boundedness of the $W_{0}^{1, p}$-norm of $u_{\epsilon}-\tilde{u}$ with respect to $\epsilon$.

In the following proposition we claim that the $L^{q}$ norms of $D u_{\epsilon}$ are locally bounded with respect to $\epsilon$. Precisely, we will prove the stronger result that the $L^{s}$-norms of $D u_{\epsilon}$ are locally bounded, with $q<s<p \frac{n}{n-\gamma}$.

Proposition 3.2. Assume that (H1)-(H3) hold with $q<p \frac{n+\gamma}{n}$, and consider $\tilde{u} \in W^{1, \frac{p(q-1)}{p-1}}(\Omega)$. Let $u_{\epsilon} \in \tilde{u}+W_{0}^{1, q}(\Omega)$ satisfy $(2.5)$, with $0<\epsilon<1$. For all $s \in$ $\left[q, p \frac{n}{n-\gamma}\right)$ there exist $\sigma, \tau$ positive and independent of $\epsilon$, such that for all $B_{R}\left(x_{0}\right) \Subset \Omega$, with $R \leq 1$, and for all $\rho<R$, we have that

$$
\int_{B_{\rho}\left(x_{0}\right)}\left|D u_{\epsilon}(x)\right|^{s} d x \leq \frac{c_{1}}{(R-\rho)^{\tau}}\left(\int_{B_{R}\left(x_{0}\right)}\left(1+\left|D u_{\epsilon}(x)\right|\right)^{p} d x\right)^{\sigma}+c_{2} R^{n},
$$

with positive constants $c_{1}, c_{2}$ independent of $\epsilon$.

Proof. From now on, we write $u$ in place of $u_{\epsilon}$. Consider $B_{R}\left(x_{0}\right) \Subset \Omega, \rho \leq r<$ $d \leq R \leq 1$, and define $\eta \in C_{c}^{\infty}(\Omega), \operatorname{supp} \eta \subseteq B_{\frac{d+r}{2}}\left(x_{0}\right), \eta \equiv 1$ in $B_{r}$ and $|D \eta| \leq \frac{4}{d-r}$. Let $\varphi=\tau_{s,-h}\left(\eta^{2} \tau_{s, h} u\right)$ with $|h|<\frac{d-r}{2}$. Then (2.5) implies

$$
\sum_{\alpha, i} \int_{\Omega} \eta^{2} \tau_{s, h}\left(A_{\epsilon, i}^{\alpha}(x, D u)\right) \tau_{s, h} u_{x_{i}}^{\alpha} d x=-\sum_{\alpha, i} \int_{\Omega} \tau_{s, h}\left(A_{\epsilon, i}^{\alpha}(x, D u)\right) 2 \eta \eta_{x_{i}} \tau_{s, h} u^{\alpha} d x
$$

We now proceed by steps.

Step 1. We prove that

$$
\int_{B_{\frac{d+r}{2}}} \eta^{2}\left|\tau_{s, h}\left(\left(|D u|^{2}\right)^{\frac{p-2}{4}} D u\right)\right|^{2} d x \leq \frac{c}{d-r} \int_{B_{d}}(1+|D u|)^{q} d x|h|^{\gamma}
$$

where the constant $c$ is independent of $\epsilon$. 
Indeed, since

$$
\begin{aligned}
& \left.\sum_{\alpha, i} \int_{\Omega} \eta^{2} \tau_{s, h}\left(A_{\epsilon, i}^{\alpha}(x, D u)\right)\right) \tau_{s, h} u_{x_{i}}^{\alpha} d x \\
& =\sum_{\alpha, i} \int_{\Omega} \eta^{2}\left(A_{\epsilon, i}^{\alpha}\left(x+h e_{s}, D u\left(x+h e_{s}\right)-A_{\epsilon, i}^{\alpha}\left(x, D u\left(x+h e_{s}\right)\right)\right) \tau_{s, h} u_{x_{i}}^{\alpha} d x\right. \\
& \quad+\sum_{\alpha, i} \int_{\Omega} \eta^{2}\left(A_{\epsilon, i}^{\alpha}\left(x, D u\left(x+h e_{s}\right)\right)-A_{\epsilon, i}^{\alpha}(x, D u(x))\right) \tau_{s, h} u_{x_{i}}^{\alpha} d x
\end{aligned}
$$

then (3.3) implies

$$
\begin{aligned}
& \sum_{\alpha, i} \int_{\Omega} \eta^{2}\left(A_{\epsilon, i}^{\alpha}\left(x, D u\left(x+h e_{s}\right)\right)-A_{\epsilon, i}^{\alpha}(x, D u(x))\right) \tau_{s, h} u_{x_{i}}^{\alpha} d x \\
& =-\sum_{\alpha, i} \int_{\Omega} \tau_{s, h}\left(A_{\epsilon, i}^{\alpha}(x, D u)\right) 2 \eta \eta_{x_{i}} \tau_{s, h} u^{\alpha} d x \\
& \quad-\sum_{\alpha, i} \int_{\Omega} \eta^{2}\left(A_{\epsilon, i}^{\alpha}\left(x+h e_{s}, D u\left(x+h e_{s}\right)\right)-A_{\epsilon, i}^{\alpha}\left(x, D u\left(x+h e_{s}\right)\right)\right) \tau_{s, h} u_{x_{i}}^{\alpha} d x
\end{aligned}
$$

By Lemma 2.5,

$$
\left|\tau_{s, h}\left(\left(|D u|^{2}\right)^{\frac{p-2}{4}} D u\right)\right|^{2} \leq c(n, m, p)\left(|D u(x)|^{2}+\left|D u\left(x+h e_{s}\right)\right|^{2}\right)^{\frac{p-2}{2}}\left|\tau_{s, h} D u(x)\right|^{2} .
$$

Therefore, using (2.4), there exists a positive constant $c$, depending on $n, m, p$ and $\nu$, such that

$$
\left|\tau_{s, h}\left(\left(|D u|^{2}\right)^{\frac{p-2}{4}} D u\right)\right|^{2} \leq c \sum_{\alpha, i}\left[A_{\epsilon, i}^{\alpha}\left(x, D u\left(x+h e_{s}\right)\right)-A_{\epsilon, i}^{\alpha}(x, D u(x))\right] \tau_{s, h} u_{x_{i}}^{\alpha} .
$$

This inequality, together with (3.4), implies

$$
\begin{aligned}
& \frac{1}{c} \int_{\Omega} \eta^{2}\left|\tau_{s, h}\left\{\left(|D u|^{2}\right)^{\frac{p-2}{4}} D u\right\}\right|^{2} d x \leq-\sum_{\alpha, i} \int_{\Omega} \tau_{s, h}\left(A_{\epsilon, i}^{\alpha}(x, D u)\right) 2 \eta \eta_{x_{i}} \times \tau_{s, h} u^{\alpha} d x \\
& \quad-\sum_{\alpha, i} \int_{\Omega} \eta^{2}\left(A_{\epsilon, i}^{\alpha}\left(x+h e_{s}, D u\left(x+h e_{s}\right)\right)-A_{\epsilon, i}^{\alpha}\left(x, D u\left(x+h e_{s}\right)\right)\right) \tau_{s, h} u_{x_{i}}^{\alpha} d x \\
& =: I_{1}+I_{2} .
\end{aligned}
$$

Let us estimate $I_{1}$. By $(2.3)$

$$
\begin{aligned}
\left|I_{1}\right| & \leq c \int_{\Omega} \eta|D \eta|\left(1+|D u(x)|+\left|D u\left(x+h e_{s}\right)\right|\right)^{q-1}\left|\tau_{s, h} u\right| d x \\
& \leq \frac{c}{d-r}\left\{\int_{B_{\frac{r+d}{2}}}\left(1+|D u(x)|+\left|D u\left(x+h e_{s}\right)\right|\right)^{q} d x\right\}^{\frac{q-1}{q}}\left\{\int_{B_{\frac{r+d}{2}}}\left|\tau_{s, h} u\right|^{q} d x\right\}^{\frac{1}{q}} .
\end{aligned}
$$


Then, using Lemma 2.3 and taking into account that $|h|<1$, we get

$$
\begin{aligned}
\left|I_{1}\right| & \leq \frac{c}{d-r}\left\{\int_{B_{d}}(1+|D u(x)|)^{q} d x\right\}^{\frac{q-1}{q}}\left\{\int_{B_{d}}\left|u_{x_{s}}\right|^{q} d x\right\}^{\frac{1}{q}}|h| \\
& \leq \frac{c}{d-r} \int_{B_{d}}(1+|D u(x)|)^{q} d x|h|^{\gamma} .
\end{aligned}
$$

Let us estimate $I_{2}$. By Hölder continuity of $A(\cdot, z)$ in (H3),

$$
\begin{aligned}
\left|I_{2}\right| & \leq c \int_{\Omega} \eta^{2}\left(1+\left|D u\left(x+h e_{s}\right)\right|\right)^{q-1}\left|\tau_{s, h} D u\right| d x|h|^{\gamma} \\
& \leq c \int_{B_{\frac{r+d}{2}}}\left(1+|D u(x)|+\left|D u\left(x+h e_{s}\right)\right|\right)^{q} d x|h|^{\gamma} \leq c \int_{B_{d}}(1+|D u|)^{q} d x|h|^{\gamma} .
\end{aligned}
$$

Thus, collecting (3.5), (3.6) and (3.7), the claim follows.

Step 2. Since $\frac{q}{p}<\frac{n+\gamma}{n}$, then we can choose $0<\theta<\gamma$ such that $\frac{q}{p}<\frac{n+\theta}{n}$. Such a choice is obtained when $0 \leq n\left(\frac{q}{p}-1\right)<\theta<\gamma$. We claim that $|D u| \in L_{\text {loc }}^{q \delta}(\Omega)$, where $\delta=\frac{p}{q} \frac{n}{n-\theta}$. Notice that $p<q \delta<p \frac{n}{n-\gamma}$.

Indeed, fixed a ball $B_{R}\left(x_{0}\right) \Subset \Omega$, with $\rho \leq r<d \leq R \leq 1$, by Step 1 and by Lemma 2.4, applied with $b=\frac{\theta}{2}$, we have $|D u|^{\frac{p}{2}} \in L^{\frac{2 n}{n-\theta}}\left(B_{r}\right)$. Moreover,

$$
\begin{aligned}
& \left\||D u|^{\frac{p}{2}}\right\|_{L^{\frac{2 n}{n-\theta}\left(B_{r}\right)}} \\
& \leq \frac{c}{(d-r)^{\theta+\gamma+2}}\left\{\left(\frac{1}{d-r} \int_{B_{d}}(1+|D u(x)|)^{q} d x\right)^{\frac{1}{2}}+\left(\int_{B_{d}}|D u(x)|^{p} d x\right)^{\frac{1}{2}}\right\}
\end{aligned}
$$

with $c$ independent of $\epsilon$. Therefore, if we set $\alpha=\left(\theta+\gamma+\frac{5}{2}\right) \frac{2 n}{n-\theta}$ the above inequality gives

$$
\int_{B_{r}}|D u(x)|^{q \delta} d x \leq \frac{\bar{c}}{(d-r)^{\alpha}}\left(\int_{B_{d}}(1+|D u(x)|)^{q} d x\right)^{\frac{q \delta}{p}} .
$$

The arbitrariness of $r, d$ and $x_{0}$ allows to conclude.

If $q=p$ then we go to Step 5. If $q>p$ we need Steps 3 and 4 .

Step 3. Since $\frac{q}{p}<\frac{n+\theta}{n}$ then $\frac{\delta q}{p}<\frac{\delta q-p}{q-p}$. In this step we prove that there exist positive constants $t, \sigma, c$, all independent of $\epsilon$, satisfying $\frac{\delta q}{p}<t \leq \frac{\delta q-p}{q-p}, 0<\sigma<1$, such that

$$
\begin{aligned}
& \int_{B_{r}}|D u(x)|^{q \delta} d x \\
& \leq \frac{c}{(d-r)^{\frac{\alpha}{1-\sigma}}}\left(\int_{B_{d}}(1+|D u(x)|)^{p} d x\right)^{\frac{(t-1) \sigma}{1-\sigma}}+\frac{1}{2} \int_{B_{d}}|D u(x)|^{q \delta} d x+c R^{n} .
\end{aligned}
$$

Indeed, $t \in\left(\frac{\delta q}{p}, \frac{\delta q-p}{q-p}\right]$ implies

$$
1<\frac{q \delta}{p}<t, \quad q \frac{t-\delta}{t-1} \leq p .
$$


By Hölder inequality

$$
\begin{aligned}
& \left(\int_{B_{d}}(1+|D u(x)|)^{q} d x\right)^{\frac{q \delta}{p}}=\left(\int_{B_{d}}(1+|D u(x)|)^{\frac{q \delta}{t}}(1+|D u(x)|)^{q\left(1-\frac{\delta}{t}\right)} d x\right)^{\frac{q \delta}{p}} \\
& \quad \leq\left(\int_{B_{d}}(1+|D u(x)|)^{q \delta} d x\right)^{\frac{q \delta}{p t}}\left(\int_{B_{d}}(1+|D u(x)|)^{q \frac{t-\delta}{t-1}} d x\right)^{\frac{q \delta}{p t}(t-1)} .
\end{aligned}
$$

Thus, (3.8) and (3.11) imply

$$
\begin{aligned}
& \int_{B_{r}}|D u(x)|^{q \delta} d x \\
& \leq\left(\int_{B_{d}}(1+|D u(x)|)^{q \delta} d x\right)^{\sigma} \frac{\bar{c}}{(d-r)^{\alpha}}\left(\int_{B_{d}}(1+|D u(x)|)^{q b} d x\right)^{(t-1) \sigma},
\end{aligned}
$$

where $\bar{c}$ is the constant in (3.8) and

$$
\sigma:=\frac{q \delta}{p t}, \quad b:=\frac{t-\delta}{t-1}
$$

By Young inequality, we get

$$
\begin{aligned}
& \int_{B_{r}}|D u(x)|^{q \delta} d x \\
& \leq \frac{c}{(d-r)^{\frac{\alpha}{1-\sigma}}}\left(\int_{B_{d}}(1+|D u(x)|)^{q b} d x\right)^{\frac{(t-1) \sigma}{1-\sigma}}+\frac{1}{2^{q \delta}} \int_{B_{d}}(1+|D u(x)|)^{q \delta} d x .
\end{aligned}
$$

Notice that by Step 2 and (3.10) the right-hand side is finite. Since $q b \leq p$ and $(|x|+|y|)^{q \delta} \leq 2^{q \delta-1}\left(|x|^{q \delta}+|y|^{q \delta}\right)$, we get (3.9).

Step 4. By using Lemma 2.2 with $Z(r):=\int_{B_{r}}|D u(x)|^{q \delta} d x$, the inequality (3.9) implies

$$
\int_{B_{\rho}}|D u(x)|^{q \delta} d x \leq \frac{c}{(R-\rho)^{\frac{\alpha}{1-\sigma}}}\left(\int_{B_{R}}(1+|D u(x)|)^{p} d x\right)^{\frac{(t-1) \sigma}{1-\sigma}}+c R^{n} .
$$

Step 5. Notice that since $\theta$ is any number in $\left(n\left(\frac{q}{p}-1\right), \gamma\right)$, then $s:=q \delta$ can be any number in $\left(\frac{p^{2}}{2 p-q}, p \frac{n}{n-\gamma}\right)$. Note that $q \leq \frac{p^{2}}{2 p-q}$. The estimate (3.2) follows when $s$ belongs to such an interval. For $s \in\left[p, \frac{p^{2}}{2 p-q}\right]$ we use Hölder inequality and we get (3.2) also in this case.

\section{Proof of Theorem 1.1}

We are now ready to prove the existence of a weak solution to (1.1).

Proof of Theorem 1.1. For all $0<\epsilon<1$, let us define the operators $A_{\epsilon, i}^{\alpha}$ as in (2.2) and let $u_{\epsilon} \in \tilde{u}+W_{0}^{1, q}(\Omega)$ satisfy

$$
\int_{\Omega} \sum_{\alpha, i} A_{\epsilon, i}^{\alpha}\left(x, D u_{\epsilon}\right) \varphi_{x_{i}}^{\alpha} d x=0 \quad \forall \varphi \in W_{0}^{1, q}(\Omega),
$$

as in Section 3. 
Step 1. We claim that there exists $u_{0} \in \tilde{u}+W_{0}^{1, p}\left(\Omega ; \mathbf{R}^{m}\right)$ such that, up to subsequences, $D u_{\epsilon} \rightarrow D u_{0}$ strongly in $L_{\text {loc }}^{p}(\Omega)$.

By Proposition 3.1, the $W_{0}^{1, p}(\Omega)$ norm of $u_{\epsilon}-\tilde{u}$ is bounded with respect to $\epsilon$. Moreover, the estimate (3.1) and Proposition 3.2 imply that, fixed $\Omega^{\prime} \Subset \Omega, D u_{\epsilon}$ is bounded in $L^{q}\left(\Omega^{\prime} ; \mathbf{R}^{n m}\right)$ uniformly with respect to $\epsilon$. Thus, there exists $u_{0} \in$ $\tilde{u}+W_{0}^{1, p}\left(\Omega ; \mathbf{R}^{m}\right)$ such that $D u_{0} \in L_{\mathrm{loc}}^{q}\left(\Omega ; \mathbf{R}^{n m}\right)$ and, up to subsequences, $u_{\epsilon}-\tilde{u} \rightarrow u_{0}-\tilde{u}$ weakly in $W_{0}^{1, p}\left(\Omega ; \mathbf{R}^{m}\right)$ and $D u_{\epsilon} \rightarrow D u_{0}$ weakly in $L_{\text {loc }}^{q}\left(\Omega ; \mathbf{R}^{n m}\right)$. Since $q<p \frac{n+\gamma}{n}$, by Rellich Theorem we get

$$
u_{\epsilon} \rightarrow u_{0} \quad \text { in } L_{\text {loc }}^{q}\left(\Omega ; \mathbf{R}^{m}\right) .
$$

To conclude, consider $\eta \in C_{c}^{\infty}(\Omega)$ and define the test function $\varphi=\left(u_{\epsilon}-u_{0}\right) \eta$. Notice that $\varphi \in W^{1, q}\left(\Omega ; \mathbf{R}^{n m}\right)$, with $\operatorname{supp} \varphi \Subset \Omega$. By (4.1)

$$
\begin{aligned}
& \int_{\Omega} \sum_{\alpha, i} A_{\epsilon, i}^{\alpha}\left(x, D u_{\epsilon}\right)\left(u_{\epsilon, x_{i}}^{\alpha}-u_{0, x_{i}}^{\alpha}\right) \eta d x=-\int_{\Omega} \sum_{\alpha, i} A_{\epsilon, i}^{\alpha}\left(x, D u_{\epsilon}\right)\left(u_{\epsilon}^{\alpha}-u_{0}^{\alpha}\right) \eta_{x_{i}}^{\alpha} d x \\
& \leq(M+1) \int_{\Omega}\left(1+\left|D u_{\epsilon}\right|\right)^{q-1}\left|u_{\epsilon}-u_{0}\right||D \eta| d x .
\end{aligned}
$$

Notice that (2.4) implies

$$
\begin{aligned}
\left|D u_{\epsilon}-D u_{0}\right|^{p} & =\left(\left|D u_{\epsilon}-D u_{0}\right|^{2}\right)^{\frac{p-2}{2}}\left|D u_{\epsilon}-D u_{0}\right|^{2} \\
& \leq 2^{\frac{p-2}{2}}\left(\left|D u_{\epsilon}\right|^{2}+\left|D u_{0}\right|^{2}\right)^{\frac{p-2}{2}}\left|D u_{\epsilon}-D u_{0}\right|^{2} \\
& \leq \frac{2^{\frac{p-2}{2}}}{\nu} \sum_{\alpha, i}\left(A_{\epsilon, i}^{\alpha}\left(x, D u_{\epsilon}\right)-A_{\epsilon, i}^{\alpha}\left(x, D u_{0}\right)\right)\left(u_{\epsilon, x_{i}}^{\alpha}-u_{0, x_{i}}^{\alpha}\right) .
\end{aligned}
$$

Thus, (4.2) and the definition of $A_{\epsilon, i}^{\alpha}$ imply

$$
\begin{aligned}
& 2^{\frac{2-p}{2}} \nu \int_{\Omega}\left|D u_{\epsilon}-D u_{0}\right|^{p} \eta d x \leq \int_{\Omega} \sum_{\alpha, i}\left(A_{\epsilon, i}^{\alpha}\left(x, D u_{\epsilon}\right)-A_{\epsilon, i}^{\alpha}\left(x, D u_{0}\right)\right)\left(u_{\epsilon, x_{i}}^{\alpha}-u_{0, x_{i}}^{\alpha}\right) \eta d x \\
& \leq(M+1) \int_{\Omega}\left(1+\left|D u_{\epsilon}\right|\right)^{q-1}\left|u_{\epsilon}-u_{0}\right||D \eta| d x-\int_{\Omega} \sum_{\alpha, i} A_{i}^{\alpha}\left(x, D u_{0}\right)\left(u_{\epsilon, x_{i}}^{\alpha}-u_{0, x_{i}}^{\alpha}\right) \eta d x \\
& \quad-\epsilon \int_{\Omega} \sum_{\alpha, i}\left|u_{0, x_{i}}^{\alpha}\right|^{q-2} u_{0, x_{i}}^{\alpha}\left(u_{\epsilon, x_{i}}^{\alpha}-u_{0, x_{i}}^{\alpha}\right) \eta d x .
\end{aligned}
$$

Thus, passing to the limit, as $\epsilon$ goes to 0 we get that the right hand side goes to 0 . We have so proved that

$$
D u_{\epsilon} \rightarrow D u_{0} \quad \text { in } L_{\mathrm{loc}}^{p}(\Omega) .
$$

Step 2. We claim that, up to subsequences, $A_{\epsilon, i}^{\alpha}\left(x, D u_{\epsilon}\right)$ converges to $A_{i}^{\alpha}\left(x, D u_{0}\right)$ in $L_{\text {loc }}^{1}(\Omega)$.

Indeed, for all $\Omega^{\prime} \Subset \Omega$, adding and subtracting $A_{i}^{\alpha}\left(x, D u_{\epsilon}\right)$, we get

$$
\begin{aligned}
& \int_{\Omega^{\prime}}\left|A_{\epsilon, i}^{\alpha}\left(x, D u_{\epsilon}\right)-A_{i}^{\alpha}\left(x, D u_{0}\right)\right| d x \\
& \leq \epsilon \int_{\Omega^{\prime}}\left|D u_{\epsilon}\right|^{q-1} d x+\int_{\Omega^{\prime}}\left|A_{i}^{\alpha}\left(x, D u_{\epsilon}\right)-A_{i}^{\alpha}\left(x, D u_{0}\right)\right| d x .
\end{aligned}
$$


By the uniform boundedness of the $L^{q}\left(\Omega^{\prime}\right)$-norms of $D u_{\epsilon}$ the first term at the right hand side goes to zero. As far as the last term is concerned, define the functions

$$
\begin{aligned}
& f_{\epsilon}(x):=\left|A_{i}^{\alpha}\left(x, D u_{\epsilon}\right)-A_{i}^{\alpha}\left(x, D u_{0}\right)\right|, \\
& g_{\epsilon}(x):=M\left\{\left(1+\left|D u_{\epsilon}(x)\right|\right)^{q-1}+\left(1+\left|D u_{0}(x)\right|\right)^{q-1}\right\} \quad \text { and } \\
& g_{0}(x):=2 M\left(1+\left|D u_{0}(x)\right|\right)^{q-1} .
\end{aligned}
$$

By (H2) we get that $f_{\epsilon}(x) \leq g_{\epsilon}(x)$ for a.e. $x \in \Omega$. Moreover, by what previously proved, $g_{\epsilon} \rightarrow g_{0}$ almost everywhere. Let us now prove that $\int_{\Omega^{\prime}} g_{\epsilon} d x \rightarrow \int_{\Omega^{\prime}} g_{0} d x$ as $\epsilon$ goes to 0 . This easily follows by (4.3) and recalling that $q \leq p+1$. A generalization of the Lebesgue Convergence Theorem allows to conclude.

Step 3. By (4.1) we get that

$$
\int_{\Omega} \sum_{\alpha, i} A_{\epsilon, i}^{\alpha}\left(x, D u_{\epsilon}\right) \varphi_{x_{i}}^{\alpha} d x=0 \quad \forall \varphi \in W^{1, q}\left(\Omega ; \mathbf{R}^{m}\right), \operatorname{supp} \varphi \Subset \Omega .
$$

By Step 2, up to subsequences,

$$
\int_{\Omega} \sum_{\alpha, i} A_{i}^{\alpha}\left(x, D u_{0}\right) \varphi_{x_{i}}^{\alpha} d x=\lim _{\epsilon \rightarrow 0} \int_{\Omega} \sum_{\alpha, i} A_{\epsilon, i}^{\alpha}\left(x, D u_{\epsilon}\right) \varphi_{x_{i}}^{\alpha} d x=0
$$

for all $\varphi \in W^{1, q}\left(\Omega ; \mathbf{R}^{m}\right), \operatorname{supp} \varphi \Subset \Omega$. Therefore, $u_{0}$ is a weak solution to (1.1).

\section{Local boundedness of the weak solutions}

Let us assume that the functions $A_{i}^{\alpha}$ in (1.1) are such that

$$
A_{i}^{\alpha}(x, z):=\sum_{j=1}^{n} a_{i j}(x, z) z_{j}^{\alpha}
$$

where $a_{i j}: \Omega \times \mathbf{R}^{n m} \rightarrow \mathbf{R}$ are Carathéodory functions such that

$$
\sum_{i, j=1}^{n} a_{i j}(x, z) \lambda_{i} \lambda_{j} \geq 0 \quad \forall \lambda \in \mathbf{R}^{n}, \forall z \in \mathbf{R}^{n m} \text { and a.e. } x \in \Omega .
$$

Let us consider the corresponding system, that is

$$
\sum_{i=1}^{n} \frac{\partial}{\partial x_{i}}\left(\sum_{j=1}^{n} a_{i j}(x, D u(x)) u_{x_{j}}^{\alpha}(x)\right)=0, \quad \alpha=1, \ldots, m .
$$

The following boundedness result is consequence of Theorem 4.1 in [10].

Theorem 5.1. Assume (5.1), (5.2) and

$$
\sum_{\alpha=1}^{m} \sum_{i=1}^{n}\left[A_{i}^{\alpha}(x, z)-A_{i}^{\alpha}(x, \tilde{z})\right]\left[z_{i}^{\alpha}-\tilde{z}_{i}^{\alpha}\right] \geq 0 \quad \forall z, \tilde{z} \in \mathbf{R}^{n m} \text { and a.e. } x \in \Omega .
$$

Assume also

$$
\nu|z|^{p}-c_{1} \leq \sum_{\alpha=1}^{m} \sum_{i=1}^{n} A_{i}^{\alpha}(x, z) z_{i}^{\alpha} \leq c_{2}(1+|z|)^{q} \quad \forall z, \tilde{z} \in \mathbf{R}^{n m} \text { and a.e. } x \in \Omega,
$$

for some $\nu, c_{1}, c_{2}>0$, with $1<p \leq q$, and also $q<p \frac{n-1}{n-p}$ if $p<n$. 
Let $u \in W_{\text {loc }}^{1, q}(\Omega)$ be a weak solution to $(5.3)$, i.e.,

$$
\int_{\Omega} \sum_{\alpha=1}^{m} \sum_{i=1}^{n} A_{i}^{\alpha}(x, D u) \varphi_{x_{i}}^{\alpha}(x) d x=0, \quad \forall \varphi \in W^{1, q}\left(\Omega ; \mathbf{R}^{m}\right), \quad \operatorname{supp} \varphi \Subset \Omega .
$$

Then $u \in L_{\mathrm{loc}}^{\infty}\left(\Omega ; \mathbf{R}^{m}\right)$.

Proof. It is easy to check that the proof of Theorem 4.1 in [10] works using (5.2) and (5.4) instead of the following condition assumed there:

$$
\sum_{i, j=1}^{n} a_{i j}(x, z) \lambda_{i} \lambda_{j} \geq M_{1} \sum_{i=1}^{n} \lambda_{i}^{2}\left(\sum_{\alpha=1}^{m}\left|z_{i}^{\alpha}\right|^{2}\right)^{\frac{p-2}{2}},
$$

for all $z \in \mathbf{R}^{m n}, \lambda \in \mathbf{R}^{n}$ and with $M_{1}>0$.

By this result and Theorem 1.1, we obtain the following existence and regularity theorem.

Theorem 5.2. Assume that (H1)-(H3) hold, with $2 \leq p \leq q<p \frac{n+\gamma}{n}$. Let $A_{i}^{\alpha}$ be as in (5.1), with $\left(a_{i j}\right)$ satisfying (5.2). For every $\tilde{u} \in W^{1, p \frac{q-1}{p-1}}\left(\Omega ; \mathbf{R}^{m}\right)$ there exists a weak solution $u$ to the Dirichlet problem (1.1), such that $u$ is locally bounded.

Proof. The existence follows by Theorem 1.1 and the local boundedness follows by Theorem 5.1 .

We conclude with two applications of the above result.

Example 5.3. Let us consider

$$
A_{i}^{\alpha}(x, z):=2 z_{i}^{\alpha}+a(x) q|z|^{q-2} z_{i}^{\alpha},
$$

where $2 \leq q, a \in C^{0, \gamma}(\bar{\Omega}), a(x) \geq 0,0<\gamma \leq 1$. The assumptions of Theorems 1.1 and 5.2 are satisfied with $p=2$. Therefore, if $q<2 \frac{n+\gamma}{n}$ and $\tilde{u} \in W^{1,2(q-1)}\left(\Omega ; \mathbf{R}^{m}\right)$ then there exists a locally bounded weak solution to the Dirichlet problem (1.1). Notice that (5.5) comes from the Euler equation of the functional

$$
\int_{\Omega}\left(|D u(x)|^{2}+a(x)|D u(x)|^{q}\right) d x .
$$

Example 5.4. Let us consider

$$
A_{i}^{\alpha}(x, z):=p(x)\left(1+|z|^{2}\right)^{\frac{p(x)-2}{2}} z_{i}^{\alpha},
$$

with $p \in C^{0, \gamma}(\bar{\Omega}), 0<\gamma \leq 1$. Assume that $2 \leq p_{1}$ and $p_{2}<p_{1} \frac{n+\gamma}{n}$, where

$$
p_{1}:=\min \{p(x)\} \quad \text { and } \quad p_{2}:=\max \{p(x)\} \text {. }
$$

The assumptions of Theorems 1.1 and 5.2 are satisfied for $p=p_{1}$ and any $q$ with $p_{2}<q<p_{1} \frac{n+\gamma}{n}$.

Therefore, if $\tilde{u} \in W^{1, \frac{p_{1}(q-1)}{p_{1}-1}}\left(\Omega ; \mathbf{R}^{m}\right)$ then there exists a locally bounded weak solution to the Dirichlet problem (1.1). Notice that (5.6) comes from the Euler equation of the functional

$$
\int_{\Omega}\left(1+|D u(x)|^{2}\right)^{\frac{p(x)}{2}} d x .
$$




\section{References}

[1] Bildhauer, M., and M. Fuchs: $C^{1, \alpha}$-solutions to non-autonomous anisotropic variational problems. - Calc. Var. Partial Differential Equations 24, 2005, 309-340.

[2] Bildhauer, M., and M. Fuchs: Higher integrability of the gradient for vectorial minimizers of decomposable variational integrals. - Manuscripta Math. 123, 2007, 269-283.

[3] BREIT, D.: New regularity theorems for non-autonomous variational integrals with $(p, q)$ growth. - Calc. Var. Partial Differential Equations 44, 2012, 101-129.

[4] Browder, F.: Nonlinear monotone operators and convex sets in Banach spaces. - Bull. Amer. Math. Soc. 71, 1965, 780-785.

[5] Canale, A., A. D’Ottavio, F. Leonetti, and M. Longobardi: Differentiability for bounded minimizers of some anisotropic integrals. - J. Math. Anal. Appl. 253, 2001, 640-650.

[6] Carozza, M., J. Kristensen, and A. Passarelli di Napoli: Higher differentiability of minimizers of convex variational integrals. - Ann. Inst. H. Poincaré Anal. Non Linéaire 28, 2011, 395-411.

[7] Choz, H. J.: Interior behaviour of minimizers for certain functionals with nonstandard growth. - Nonlinear Anal. 19, 1992, 933-945.

[8] Colombo, M., and G. Mingione: Regularity for double phase variational problems. - Arch. Ration. Mech. Anal. 215, 2015, 443-496.

[9] Colombo, M., and G. Mingione: Bounded minimisers of double phase variational integrals. - Preprint, 2014.

[10] Cupini, G., P. Marcellini, and E. Mascolo: Local boundedness of solutions to quasilinear elliptic systems. - Manuscripta Math. 137, 2012, 287-315.

[11] Cupini, G., P. Marcellini, and E. Mascolo: Local boundedness of solutions to some anisotropic elliptic systems. - Contemp. Math. 595, 2013, 169-186.

[12] Cupini, G., P. Marcellini, and E. Mascolo: Existence and regularity for elliptic equations under $p, q$-growth. - Adv. Differential Equations 19, 2014, 693-724.

[13] De Giorgi, E.: Un esempio di estremali discontinue per un problema variazionale di tipo ellittico. - Boll. Unione Mat. Ital. (4) 1, 1968, 135-137.

[14] D'Ottavio, A.: A remark on the paper: "Some remarks on the regularity of minimizers of integrals with anisotropic growth". - Comment. Math. Univ. Carolin. 36, 1995, 489-491.

[15] Esposito, L., F. Leonetti, and G. Mingione: Sharp regularity for functionals with $(p, q)$ growth. - J. Differential Equations 204, 2004, 5-55.

[16] Fonseca, I., I. Malý, and G. Mingione: Scalar minimizers with fractal singular sets. - Arch. Ration. Mech. Anal. 172, 2004, 295-307.

[17] Giaquinta, M.: Growth conditions and regularity, a counterexample. - Manuscripta Math. $59,1987,245-248$.

[18] Giaquinta, M.: Multiple integrals in the calculus of variations and nonlinear elliptic systems. - Ann. of Math. Stud. 105, Princeton Univ. Press, 1983.

[19] Giusti, E.: Direct methods in the calculus of variations. - World Scientific Publishing Co., Inc., River Edge, NJ, 2003.

[20] Hartman, P., and G. Stampacchia: On some non-linear elliptic differential-functional equations. - Acta Math. 115, 1966, 271-310.

[21] Hong, M.-C.: Some remarks on the minimizers of variational integrals with nonstandard growth conditions. - Boll. Unione Mat. Ital. A 6, 1992, 91-101.

[22] Leonetti, F.: Weak differentiability for solutions to nonlinear elliptic systems with $p, q$-growth conditions. - Ann. Mat. Pura Appl. 162, 1992, 349-366. 
[23] Leonetti, F., and E. Mascolo: Local boundedness for vector valued minimizers of anisotropic functionals. - Z. Anal. Anwend. 31, 2012, 357-378.

[24] Leray, J., and J. L. Lions: Quelques résultats de Višik sur les problèmes elliptiques nonlinéaires par les méthodes de Minty-Browder. - Bull. Soc. Math. France 93, 1965, 97-107.

[25] Marcellini, P: Un example de solution discontinue d'un problème variationnel dans le cas scalaire. - Preprint 11, Istituto Matematico "U. Dini", Università di Firenze, 1987.

[26] Marcellini, P.: Regularity of minimizers of integrals in the calculus of variations with nonstandard growth conditions. - Arch. Ration. Mech. Anal. 105, 1989, 267-284.

[27] Marcellini, P.: Regularity and existence of solutions of elliptic equations with $p, q$-growth conditions. - J. Differential Equations 90, 1991, 1-30.

[28] Mingione, G.: Regularity of minima: an invitation to the dark side of the calculus of variations. - Appl. Math. 51, 2006, 355-426.

[29] Mingione, G.: Singularities of minima: a walk on the wild side of the calculus of variations. - J. Global Optim. 40, 2008, 209-223.

[30] SverÁK, V., and X. YAN: Non-Lipschitz minimizers of smooth uniformly convex functionals. - Proc. Natl. Acad. Sci. USA 99:24, 2002, 15269-15276.

Received 24 November 2014 • Accepted 8 December 2014 